\title{
ALGEBRAIC CYCLES AND INTERSECTION HOMOLOGY SHOJI YOKURA
}

(Communicated by William C. Waterhouse)

\begin{abstract}
We consider Dubson's conjecture that the fundamental class in homology of an algebraic cycle on a complex algebraic variety is the image of a middle intersection homology class. In the case when the variety has only isolated singularities, we prove it for rational coefficients, and we give a counterexample to it for integral coefficients
\end{abstract}

Introduction. Intersection homology theory, abbreviated $\mathrm{IH}_{*}^{\bar{p}}$, was developed by M. Goresky and R. MacPherson [GM1, GM2, GM3] for the study of singular spaces, introducing the concept of perversity $\bar{p}$, which, roughly speaking, gives some geometric conditions on how chains enter the singularities of the singular space. In this paper we consider the intersection homology group $\mathrm{IH}_{*}^{\bar{m}}(X)$ of complex varieties with the middle perversity $\bar{m}=(0,0,1,1,2, \ldots)$ and rational coefficients $\mathbf{Q}$ or integral coefficients $\mathbf{Z}$. For the sake of simplicity we drop the symbol $\bar{m}$.

Some time back A. S. Dubson conjectured that the fundamental class in homology of an algebraic cycle on a complex algebraic variety is the image of a middle intersection homology class. (His conjecture (or problem) is imprecisely stated in J. L. Brylinski's Séminaire Bourbaki article [Bry, §5 Problèmes ouverts].) Given an algebraic cycle $V$ of dimension $k$ in an algebraic variety $X$, we can define its fundamental class $[V] \in H_{2 k}(X)$. So, Dubson's conjecture is

$$
[V] \in \operatorname{Image}\left(\operatorname{IH}_{2 k}(X) \rightarrow H_{2 k}(X)\right)
$$

where $\mathrm{IH}_{*}(X) \rightarrow H_{*}(X)$ is the natural (or canonical) homomorphism.

In this paper we restrict ourselves to singular complex varieties with isolated singularities and consider Dubson's conjecture above.

I would like to thank Clint McCrory and Robert Varley for their useful comments and suggestions, and the referee for some suggestions.

2. A basic fact. For details and basic facts of intersection homology theory, see serial papers of Goresky and MacPherson [GM1, GM2, GM3]. In general it may not be easy to compute $\mathrm{IH}_{*}(X)$ (with $\mathbf{Z}$ or $\mathbf{Q}$ as coefficients). However, if $X$ has a single isolated singularity $x$ (of course we can allow many isolated singular points), then the intersection homology group is "almost" equal to the usual homology

Received by the editors April 9, 1986 and, in revised form, February 2, 1987.

1980 Mathematics Subject Classification (1985 Revision). Primary 14C15, 57N65; Secondary $14 \mathrm{~F} 99$ 
group as in the following proposition:

PROPOSITION [GM1]. Let $X$ be an $n$-dimensional singular complex variety which has a single isolated singular point $x$. Then

$$
\mathrm{IH}_{i}(X)=\left\{\begin{array}{l}
H_{i}(X) \quad \text { if } i>n, \\
\operatorname{Image}\left(H_{i}(X-\{x\}) \rightarrow H_{i}(X)\right) \quad \text { if } i=n, \\
H_{i}(X-\{x\}) \quad \text { if } i<n .
\end{array}\right.
$$

Using this basic proposition or taking this as the definition of $\mathrm{IH}_{*}(X)$, we will discuss Dubson's conjecture in the following section.

3. Results. First of all we take the rational coefficients $\mathbf{Q}$ for $\mathrm{IH}_{*}(X)$.

THEOREM 1. Let $X^{n}$ be an $n$-dimensional complex algebraic variety with a single isolated singularity $p$, and let $V_{i}$ be any algebraic cycle of dimension i in $X$. Then Dubson's conjecture is true, i.e.; for any $i$,

$$
\left[V_{i}\right] \in \operatorname{Image}\left(\mathrm{IH}_{2 i}(X) \rightarrow H_{2 i}(X)\right) .
$$

REMARK 2. We can, of course, allow many isolated singularities in the theorem. But for the sake of simplicity of the proof we consider only the case when $X$ has a single isolated singularity. If we take the integral coefficients $\mathbf{Z}$ instead of $\mathbf{Q}$, then the theorem does not hold as we will see in an example given later.

PROOF OF THEOREM 1 . If $i>n / 2$, then by the Proposition above, the theorem clearly holds. So we only have to look at the case when $i \leq n / 2$. (Also we can assume that $i>0$ because for $i=0$ the theorem evidently holds.) Let $f: \tilde{X} \rightarrow X$ be a resolution (by finitely many blowing-ups) of $X$ and let $E=f^{-1}(p)$. Let $D_{p} \subset X$ be the set of points of distance at most $\varepsilon$ from $p$, where $\varepsilon$ is a sufficiently small positive number. Here distance is meant to be the Euclidean distance with respect to some local analytic embedding of a neighborhood of $p$ into $\mathbf{C}^{N}$. Set $M=f^{-1}\left(D_{p}\right)$, and $B=f^{-1}\left(\partial D_{p}\right)$, where $\partial D_{p}$ is the boundary of $D_{p}$, i.e., the set of points of distance exactly $\varepsilon$ from $p$. Then we have the following commutative diagram with rows being exact:

$$
\begin{aligned}
& \cdots \rightarrow H_{2 i}(\tilde{X}-E) \stackrel{i_{*}}{\rightarrow} H_{2 i}(\tilde{X}) \stackrel{\tilde{j}_{*}}{\rightarrow} H_{2 i}(\tilde{X}, \tilde{X}-E) \rightarrow \cdots
\end{aligned}
$$

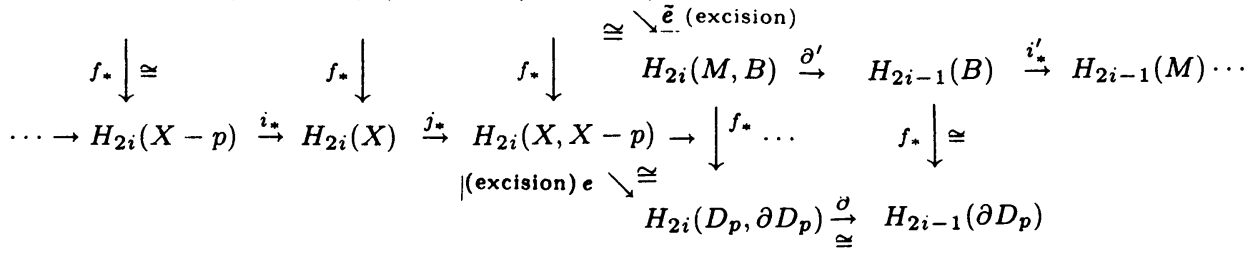

In the diagram, $e$ and $\tilde{e}$ are excision isomorphisms. Since $D_{p}$ is contractible, $H_{j}\left(D_{p}\right)=0$ for $j>0$, so $\partial: H_{2 i}\left(D_{p}, \partial D_{p}\right) \stackrel{\cong}{\rightarrow} H_{2 i-1}\left(\partial D_{p}\right)$ is an isomorphism. Now if we can show

$$
j_{*}([V])=0,
$$

then we get the statement of the theorem. Thus all we have to do is to show (1). (1) is equivalent to

$$
\partial \circ e \circ j_{*}([V])=0
$$


If we take a proper transform $\tilde{V}$ of $V$ under $f$, then we have $f_{*}([\tilde{V}])=[V]$. So by the above commutative diagram, $(2)$ is equivalent to

$$
\partial^{\prime} \circ \tilde{e} \circ \tilde{j}_{*}([\tilde{V}])=0 .
$$

Here we note that $2 i-1 \leq n-1<n$. Also we recall the following useful fact from Goresky and MacPherson's lecture notes [GM3]:

FACT (COROLLARY 1 OF [GM3, §3]). Let the situation be as above and let $K$ be the kernel of $i_{*}^{\prime}: H_{*}(B) \rightarrow H_{*}(M)$. Then we have

$$
K=H_{n}(B) \oplus H_{n+1}(B) \oplus \cdots \oplus H_{2 n-1}(B) .
$$

In other words, this fact can be interpreted as that $i_{*}^{\prime}: H_{s}(B) \rightarrow H_{s}(M)$ is injective if $s<n$. Thus, since $2 i-1<n$ in our case, we can conclude that $i_{*}^{\prime}$ in the diagram above is injective, which means that $\partial^{\prime}$ in the diagram is a zero map. Therefore we can conclude the identity (3) above. This completes the proof of the theorem.

REMARK 3. One of typical examples of complex algebraic varieties with an isolated singularity is the projective cone $X^{n}=c\left(Y^{n-1}\right) \subset \mathbf{P}^{N+1}$ over a nonsingular projective variety $Y^{n-1} \subset \mathbf{P}^{N}$. In this case, the injectivity of $i_{*}^{\prime}: H_{s}(B) \rightarrow H_{s}(M)$ for $s<n$ can be shown by using a standard technique such as the Hard Lefschetz Theorem etc. In fact, the above theorem is motivated by observing the theorem in the case of projective cones.

Next, if we take the integer coefficients $\mathbf{Z}$ for $\mathrm{IH}_{*}(X)$, then surely Theorem 1 does not hold as we pointed out in Remark 2 . Here we give a simple interesting example, which is the projective cone over a nonsingular projective variety. Since we take a double point cycle as our algebraic cycle, we discuss a little about a generic projection and double point locus. Let $V^{k}$ be a $k$-dimensional projective algebraic variety of degree $d$ in $\mathbf{P}^{N}(N>k)$. Then for any integer $r \geq k$, there exists a $(N-r-1)$-dimensional linear subspace $H_{r}$ in $\mathbf{P}^{N}$ such that $V^{k} \cap H_{r}=\varnothing$. Suppose that $H_{r}$ is a linear subspace of $\mathbf{P}^{N}$ cut out by $r+1$ independent linear forms $L_{0}, \ldots, L_{r}$. Then we define a map: $\mathbf{P}^{N}-H_{r} \rightarrow \mathbf{P}^{r}$ by

$$
x \mapsto\left(L_{0}(x) ; \ldots ; L_{r}(x)\right), \quad \text { or } \quad x \mapsto \operatorname{Span}\left(H_{r}, x\right) \cap \mathbf{P}^{r} .
$$

The induced map obtained by the restriction of this map to $V^{k}$ is called the projection from the center $H_{r}$, and denoted by $\pi_{r}$. Here we note that if $k=r$, then $\pi_{k}: V^{k} \rightarrow \mathbf{P}^{k}$ is a $d$-sheeted branched cover of $\mathbf{P}^{k}$, where $\operatorname{deg} V=d$ (cf. [GH, p. 168]). The double point locus $D\left(\pi_{r}\right)$ of $\pi_{r}$ is defined by

$$
D\left(\pi_{r}\right)=\text { The closure of }\left\{x \in V \mid \exists y \in V, y \neq x \text { such that } \widehat{x y} \cap H_{r} \neq \varnothing\right\},
$$

where $\widehat{x y}$ denotes the line through $x$ and $y$. It is well known (cf. [Jo]) that all components of $D\left(\pi_{r}\right)$ are irreducible and hence have dimension $2 k-r$. Before stating our next theorem (example) we observe the following: If $D\left(\pi_{r}\right)$ is the double point locus of a generic projection from the projective cone $c\left(V^{n}\right)$ in $\mathbf{P}^{N+1}$ with the center $H_{r}$, then $D\left(\pi_{r}\right)$ is the projective cone over the double point locus $D\left(\pi_{r-1}\right)$ of $\pi_{r-1}: V \rightarrow \mathbf{P}^{r-1}$, which is the restriction of $\pi_{r}$ to the base space $V$.

THEOREM 4. Let $V^{1}$ be a nonsingular projective algebraic curve of degree $d$ in $\mathbf{P}^{3}$ and let $X^{2}=c(V)$ be the projective cone over $V$ in $\mathbf{P}^{4}$ and $\pi_{3}: c(V) \rightarrow \mathbf{P}^{3}$ be a generic projection from a point. Then the double point class $\left[D\left(\pi_{3}\right)\right]$ is in 
$\mathrm{IH}_{2}(c(V))$ if and only if $\chi(V) \equiv 0(\bmod d)$, where $\chi(V)$ is the topological EulerPoincaré characteristic of $V$.

Proof. First look at the double point locus of $\pi_{2}: V \rightarrow \mathbf{P}^{2}$. It is well known (e.g. see [Mum]) that $\pi_{2}(V)$ has ordinary double points, i.e., nodes as singular points and that the number of nodes is $(d-1)(d-2) / 2-g(V)$, where $g(V)$ is the topological genus of $V$. Hence $D\left(\pi_{2}\right)$ consists of $(d-1)(d-2)-2 g(V)$, i.e., $\left(d^{2}-3 d+\chi(V)\right)$ points. So the double point locus $D\left(\pi_{3}\right)$ is topologically the bouquet of $\left(d^{2}-3 d+\chi(V)\right) 2$-spheres $S^{2}, S^{2} \vee \cdots \vee S^{2}$.

Now consider the following diagram, where $c$ denotes the cone point of $X$ :

$$
\begin{aligned}
& \rightarrow H_{2}(X-\{c\}) \stackrel{i_{*}}{\rightarrow} \quad H_{2}(X) \stackrel{j_{*}}{\rightarrow} \quad H_{2}(X, X-\{c\}) \\
& \psi \quad \| l e \text { (excision) } \\
& {\left[D\left(\pi_{3}\right)\right] \quad H_{2}(D, \partial D) \cong H_{1}(\partial D)}
\end{aligned}
$$

where we use the same notation (or symbol) as before.

Claim. $\left[D\left(\pi_{3}\right)\right] \in \mathrm{IH}_{2}(X ; \mathbf{Z})=$ Image $i_{*}$ if and only if $\chi(V) \equiv 0(\bmod d)$.

All we have to do is to see when $\partial \circ e \circ j_{*}\left(\left[D\left(\pi_{3}\right)\right]\right)=0$. First we show the following

LEMMA A. $H_{1}(\partial D) \cong H_{1}(V) \oplus \mathbf{Z} / d$.

ProOf. Blow up $X$ along the cone point $c$. Then, using the same symbols ( $M$ and $B$ ) used in the proof of Theorem 1 and the same notation as in Milnor's book [Mi, §12], $M$ can be (homotopically) considered as the total space $E(\xi)$ of the associated line bundle $\xi$ over $V$ and $B$ can be (homotopically) considered as the total space $E_{0}(\xi)$ of the subbundle of $\xi$ whose fiber is $\mathbf{C}-\{0\}$. Then we have the following Gysin sequence:

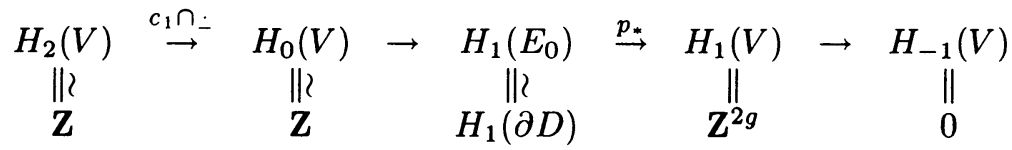

where $c_{1}=c_{1}(\xi)$ is the first Chern class of the line bundle $\xi, p: E_{0}(\xi) \rightarrow V$ is the projection map, and $g$ is the topological genus of $V$. Since $H_{2}(V)=\mathbf{Z}$ is generated by the fundamental class $[V]$ and $c_{1} \cap[V]$ is nothing other than $[V \cap H]$, where $H$ is a generic hyperplane in $\mathbf{P}^{3}$, which is by definition, the degree of $V$, i.e., $d$. Therefore, since $H_{0}(V)=\mathbf{Z}$, the map $c_{1} \cap_{-}: H_{2}(V) \rightarrow H_{0}(V)$ is just the multiplication by $d$, hence $c_{1} \cap_{-}$is injective. Since $\mathbf{Z}^{2 g}$ is free, we have

$$
H_{1}\left(E_{0}\right)=H_{1}(\partial D) \cong H_{1}(V) \oplus \mathbf{Z} / d .
$$

That is, any class supported on the fiber is a torsion element.

Recall that $D\left(\pi_{3}\right)$ is the bouquet of $\left(d^{2}-3 d+\chi(V)\right) 2$-spheres $S^{2}$ 's. Then $\partial \circ e \circ j_{*}\left(\left[D\left(\pi_{3}\right)\right]\right)$ is supported on the disjoint union of 1 -spheres $S^{1}$ 's over $\left(d^{2}-3 d+\chi(V)\right)$ points in $V$. Since we can have a simple curve (i.e., a contractible curve) going through all these points only once, we can say that $\partial \circ e \circ j_{*}\left(\left[D\left(\pi_{3}\right)\right]\right)$ is supported on $\left(d^{2}-3 d+\chi(V)\right) S^{1}$ 's at just one point. Since this is in the torsion part $\mathbf{Z} / d$ of $H_{1}(\partial D)=H_{1}(V) \oplus \mathbf{Z} / d$,

$$
\partial \circ e \circ j_{*}\left(\left[D\left(\pi_{3}\right)\right]\right)=\left(d^{2}-3 d+\chi(V)\right)_{\bmod d} .
$$


Therefore

$$
\begin{aligned}
{\left[D\left(\pi_{3}\right)\right] \in \text { Image } i_{*} } & \Leftrightarrow \partial \circ e \circ j_{*}\left(\left[D\left(\pi_{3}\right)\right]\right)=0 \\
& \Leftrightarrow\left(d^{2}-3 d+\chi(V)\right)_{\bmod d}=0 \\
& \Leftrightarrow \chi(V) \equiv 0(\bmod d) .
\end{aligned}
$$

This is what we wanted to show.

\section{REFERENCES}

[Bry] J. L. Brylinski, (C'o)-homologie d'intersection et faisceaux pervers, Séminaire Bourbaki, $34^{\mathrm{e}}$ année, no. 585, 1981/1982.

[GM1] M. Goresky and R. MacPherson, Intersection homology. I, Topology 19 (1980), 135-162.

[GM2] _ Intersection homology. II, Invent. Math. 72 (1983), 77-130.

[GM3] _ , On the topology of complex algebraic maps, Algebraic Geometry (Proceedings, La Rabida), Lecture Notes in Math., vol. 961, Springer-Verlag, Berlin and New York, 1982, 119-129.

[GH] P. Griffiths and J. Harris, Principles of algebraic geometry, Wiley, 1978.

[Jo] K. W. Johnson, Immersion and embedding of singular varieties, Acta Math. 140 (1978), 49-74.

[Mi] J. Milnor and J. Stasheff, Characteristic classes, Ann. of Math. Stud., No. 76, Princeton Univ. Press, Princeton, N.J., 1974.

[Mum] D. Mumford, Algebraic geometry. I, Complex Projective Varieties, Springer-Verlag, Berlin and New York, 1976.

FACUlty OF ENGINEERING, University OF Kagoshima, Kagoshima, 890, JAPAN 